\title{
Epidermal Studies in Identification of Jatropha Species
}

\author{
Kranti Rai ${ }^{1} \&$ Dr. Ela Tiwari ${ }^{2}$ \\ Research scholar \& Prof. Deptt. Of Botany \\ ${ }^{1,2}$ (Department Of Botany/ Government Autonomous P.G. College of Excellence/University Of Sagar MP/India)
}

\begin{abstract}
The detailed cuticular study of jatropha plant species has been carried out to help in their identification. Jatropha species are traditional plants of Euphorbiaceace family. Tropically distributed Jatropha curcas and Jatropha gossypifolia have medicinal and toxic properties due to the presence of their chemical constituents. In Jatropha curcas anomocytic, anomotetracytic, actinocytic, paratetracytic stomata, bicelled glandular hair, uniseriate glandular hair, unicelled cylindrical glandular hair, two armed cylindrical glandular hair were reported. While in this Jatropha gossypifolia has been investigation found brachyparacytic, anomotetracytic, anomocytic stomata, uniseriate aseptate flagellate glandular hair, and multiseriate capitate glandular hair. Stomata number, stomata density, stomata frequency, stomata index, epidermal number, epidermal density, epidermal frequency, trichome number, trichome density, trichome frequency, trichome index are found in my research. The various parameters which are used in my study are helpful in identifying species plays a vital role in my research. The study indicates the taxonomic utility of the different parameter of plant species.
\end{abstract}

Keywords: Second $=n d$, edition $=e d s$, figure $=$ fig, Length $=l$, breadth $=b$

\section{Introduction}

Jatropha is a tropical genus of approximately 175 succulent plants, shrubs, trees from the family Euphorbiaceous. Generally, Jatropha has been used as abortifacient and remedies for dropsy, gout, tumors, syphilis, parasitic skin infestation (Iwu, 1993). Jatropha plants contain several toxins including lectin, saponin, carcinogenic phorbol and a trypsin inhibitor. Jatropha gossypifolia is known as invasive and highly toxic to people and animals. Jatropha curcas is used as purgative oil that is toxic in large quantities (smith1923). The present paper deals to use microscopic examinations of epidermal cells, stomata and trichomes with the aim of providing useful taxonomic data that would give further insight into proper classification, delineation and identification of the studied taxa.

Abdulrahaman and et.al. $(2009,2010)$ has been discovered stomata complex in Dioscorea, Jatropha species. Patel and et.al. (1971) has been noticed anisocytic, anomocytic, diacytic, paracytic stomata and stomata with a single subsidiary cell in some polemoniales. Anomocytic stomata are found in Boerhavia species except in $B$. diffusa where a mixture of both anomocytic and anisocytic types occur. Trichomes are uniseriate and unbranched but are variable in size, distribution and abundance (Fadeyi and et.al.1989). According to Camargo and et.al.(2011) in 35 rainforest tree species in Central Amazonia, the most common stomatal type was anomocytic(37\%), followed by paracytic $(26 \%)$ and anisocytic $(11 \%)$. Stomatal studies have been done by Hameed and et.al. (2008) on some plants of Polygonaceae, Abid and et.al.(2007) on monocots within flora of Karachi, Pakistan, Ahmad and et.al.(2009) on dicot flora of a district tank in Pakistan.

Trichomes have been discovered by Sahu (1982, 83, 84, 85), Tiwari (1982), Faust and Jones (1973) and Inamdar and Gangadhara (1975). They have indicated the taxonomic utility of the morphological characters of trichomes. Therefore the present work has been undertaken, which deals with a view to their elucidating diagnostic significance.

\section{Materials and methods}

In the central India, hills of Sagar district are made from Vindhya and Basalt rocks and tropical dry deciduous forest (Champian and seth 1968). Jatropha curcas and jatropha gossypifolia are taken for the microscopic study. Epidermal structures are studied by the methods of Bobous and Beakbane (1971) under microscope and camera lucida diagrams are prepared.

There are expressed various parameter of each complex type by following formula methods:

(1) Stomata index $\%(\mathrm{SI})=\quad$ stomata density $* 100$

(Stomata density + epidermal cell density) (Salisbury, 1927).

Where, [Area of grid $=5 * 5=25$ square micron (where objective lens is $10 \mathrm{x}$ and eye lens is $15 \mathrm{x}$ )

Area of grid $=1.25 * 1.25=1.56$ square micron (where objective lens is $40 \mathrm{x}$ and eye lens is $15 \mathrm{x}$ ).] 
(2) Stomata density $=\quad$ stomata frequency

$10 *$ area of grid square micron

(3) Stomata frequency $=$ number of stomata per unit area

(4) Trichome index $\%(\mathrm{TI})=\quad$ trichome density $* 100$

(Trichome density + epidermal cell density)

Where, [Area of grid $=5 * 5=25$ square micron (where objective lens is $10 \mathrm{x}$ and eye lens is $15 \mathrm{x}$ )

Area of grid $=1.25 * 1.25=1.56$ square micron (where objective lens is $40 \mathrm{x}$ and eye lens is $15 \mathrm{x}$ ).]

(5) Trichome density = trichome frequency

$10 *$ area of gridsquare micron

(6) Trichome frequency $=$ number of trichome per unit area

Trichomes are epidermal outgrowths or appendages on plants. Trichomes are differentiated mainly in two parts,

1. Proximal foot that is lying in the epidermis.

2. Distal part body that is lying above the foot.

Nomenclature and terminology of trichome are based on Ramayya (1962) and Payne (1978).

In the stomata, terminology and nomenclature are mainly based on Metcalfe and Chalk (1950). According to Clive Anthony stace (1989) Thirty-one types of arrangement of subsidiary cells in the mature stomatal complex of vascular plants, adapted from Dilcher (1974).

According to David Frederick cutler etal (2008) five other types sunken stomata, tetracytic stomata, stomata with a single subsidiary cell, traditional between diacytic and paracytic, hemidiacytic stomata are also found in plants.

A stoma is a small aperture on the surface of land plants. It is surrounded by a pair of specialized epidermal cells called guard cells, which act as a turgor-driven valve that open and close the pores in response to given environmental conditions.

\section{Observation}

Geographically, Jatropha curcas is widely distributed in almost all countries in the tropical regions of Africa, Asia and Latin America and has several local names, showing that it has become almost indigenized in most of countries and can withstand conditions of severe drought and low soil fertility. Jatropha gossypifolia is native to Brazil and tropical America from Mexico to Paraguay and the Caribbean region. It was imported into Australia in the late 1800's, probably as a garden, ornamental and had naturalized in Queenland by 1912. It is major weed in Australia grown as live fence, and also found in waste places. It occurs throughout tropical Africa, except the dry regions in southern Africa, but including South Africa. It is widely distributed in India.

3.1. Jatropha curcas

3.1.1. Scientific classification

Order - Malpighiales

Family - Euphorbiaceous

Genus - Jatropha

Species - curcas

3.1.2. Morphology

It is a poisonous, drought-resistant perennial with smooth gray bark which excudes a whitish colored, watery, latex when cut. The leaves are green to pale green, deciduous, and alternate to sub opposite and three to five lobed with a spiral phyllotaxis. The stem is erect; $1.80-7.0 \mathrm{~m}$ branched at the top. Flowers have several too many in greenish cymes, yellowish, bell-shaped, sepals 5, broadly deltoid. Male and female flowers are produced on the same inflorescence, averaging 20 male flowers to each female flower or 10 male flowers to each female flower. The fruit is a broadly ellipsoid capsule, smooth-skinned containing three ellipsoid seeds.

\subsubsection{Trichome characters}

Trichomes plate A, Fig 1. Uniseriate capitates glandular hair: Foot compound, body differentiated into stalk and head, entire, uniseriate, head capitates, contents translucent, walls thin, smooth and straight.

Distribution: on pedicel, gynoceium.

Trichomes plate A, Fig 2, 5, 7. Unicelled flagellate glandular hair: Foot compound, body unicelled, undifferentiated, oblique, pointed apex, contents translucent, and walls thin, smooth and straight.

Distribution: on pedicel, petal, stem, androceium.

Trichomes plate A, Fig 3. Developing uniseriate flagellate glandular hair: Foot compound, body multicelled, flagellate, differentiated in stalk and head, contents transparent, walls thin, smooth and straight.

Distribution: on stem.

Trichomes plate A, Fig 9, 8. Uniseriate flagellate glandular hair: Foot compound, body multicelled, flagellate, differentiated into stalk and head, contents translucent, walls thin, smooth and straight.

Distribution: on calyx, corolla. 
Trichomes plate A, Fig 10, 6. Unicelled branched glandular hair: Foot compound, body unicelled branched entire, contents translucent, walls thin, smooth and straight.

Distribution: on calyx, corolla, stem, gynoceium.

Trichomes plate A, Fig 4. Papillate glandular hair: Foot compound, body papillose, contents translucent, walls thin, smooth and straight.

Distribution: on stem.

\subsubsection{Stomata characters}

There are following stomata that distributed on different parts of Jatropha curcas:

Anomocytic, anomotetracytic stomata:

Distribution: on leaf, stem, and pedicel.

Actinocytic stomata:

Distribution: On calyx, corolla

Paratetracytic stomata:

Distribution: on fruit wall

3.1.5. Toxic constituents

Curcin A and B, phorbolesters, phytic acid, curcinoleic acid, lectin, ricin and abrin.

3.1.6. Economic importance

The plant is useful in treatment of skin diseases and other ailments. It is wound disinfectant, purgative, rheumatism. The latex of Jatropha contains an alkaloid known as "Jatrophine" which is believed to have anticancerous properties.

\subsubsection{Toxicology}

The plant can be show mild symptoms and toxic symptoms. The important symptoms of poisoning included diarrhoea, inability to keep normal posture, depression and lateral recumbence.

3.2. Jatropha gossypifolia

3.2.1. Scientific classification

Order - Malpighiales

Family- Euphorbiaceous

Genus - Jatropha

Species - gossypifolia

\subsubsection{Morphology}

It is a busy, gregarious shrub up to $1.8 \mathrm{~m}, 3-5$ lobed, approximately $20 \mathrm{~cm}$ long and wide with leaves having a long petiole, covered with glandular hairs from the euphorbiaceous family. The stem is hairy and nonwoody. Flowers are red-crimson of purple in corymbs, with greenish seed in smooth, glabrous, oblong capsule.

\subsubsection{Trichome characters}

Trichome plate A, Fig 1. Uniseriate flagellate glandular hair: Foot compound; body uniseriate, differentiated in stalk and head; contents translucent; walls thick, smooth and straight.

Distribution: all the parts of this plant except gynoceium.

Trichomes plate A, Fig 2. Multiseriate capitates glandular hair: Foot compound; body multiseriate, differentiated in stalk and head, stalk multicelled, head capitate; contents translucent; walls thin, smooth, entire. Distribution: all the parts of this plant except gynoceium.

\subsubsection{Stomata characters}

Brachyparacytic, anomotetracytic stomata:

Distribution: on leaf, calyx.

Anomocytic stomata:

Distribution: on stem, pedicel.

Anomotetracytic stomata:

Distribution: on fruit wall

3.2.5. Toxic constituents

Ricinine, alkaloid, jatrophin. 


\subsubsection{Economic importance}

The plant is antibiotic, insecticidal and used for toothache and as blood purifier. In the Philippines, cytoplasm of fresh leaves is applied to swollen breasts. In Venezuela, roots are used in leprosy, decoction of leaves used as purgative and stomachic. The latex used on ulcers. The leaves are used as febrifuge for intermittent fevers. In Ayurveda the oil from the seeds is used for treatment of eczema and skin itches, though the main use is as renewable source of energy as biodiesel. The roots are employed against leprosy, as an antidote for snakebite and in urinary complaints. A decoction of the bark is used as an emmenagogue and leaves for stomachache, venereal disease and as blood purifier.

\subsubsection{Toxicology}

The seeds and seed oil rapidly produce abdominal pain followed by vomiting and diarrhoea when consumed in excess. The important signs of toxicity include ptosis, reduction of body weight, darkening of cuticle, abnormal pupation and hind limb paralysis.

\subsection{Graphs And Tables}

The macro morphological characters are assessed on different parts of studied plants.

4.1.1. There are presented number of stomata of studied plants in table 1

\begin{tabular}{|c|c|c|c|c|c|c|c|c|c|c|}
\hline $\begin{array}{l}\text { Name } \\
\text { of plant }\end{array}$ & $\begin{array}{l}\text { Name of } \\
\text { plant } \\
\text { parts }\end{array}$ & $\begin{array}{l}\text { Type of } \\
\text { stomata }\end{array}$ & $\begin{array}{l}\text { Num } \\
\text { ber of } \\
\text { stoma } \\
\text { ta }\end{array}$ & $\begin{array}{l}\text { Stomat } \\
\text { al } \\
\text { freque } \\
\text { ncy }\end{array}$ & $\begin{array}{l}\text { Stomat } \\
\text { al } \\
\text { density }\end{array}$ & $\begin{array}{l}\text { Type of } \\
\text { epider } \\
\text { mal cell }\end{array}$ & $\begin{array}{l}\text { Numb } \\
\text { er of } \\
\text { epider } \\
\text { mal } \\
\text { cell } \\
\end{array}$ & $\begin{array}{l}\text { Freque } \\
\text { ncy of } \\
\text { epider } \\
\text { mal } \\
\text { cell } \\
\end{array}$ & $\begin{array}{l}\text { Densit } \\
\mathrm{y} \text { of } \\
\text { epider } \\
\text { mal } \\
\text { cell } \\
\end{array}$ & $\begin{array}{l}\text { Stoma } \\
\text { tal } \\
\text { index }\end{array}$ \\
\hline $\begin{array}{l}\text { Jatroph } \\
\text { a } \\
\text { gossypif } \\
\text { olia }\end{array}$ & $\begin{array}{l}\text { Upper } \\
\text { Layer } \\
\text { Of Leaf }\end{array}$ & $\begin{array}{l}\text { Brachypara } \\
\text { cytic, } \\
\text { Anomotetr } \\
\text { acytic }\end{array}$ & $\begin{array}{l}1 \text { Or } \\
\text { Rare }\end{array}$ & 0.32 & 0.02 & $\begin{array}{l}\text { Irregula } \\
\mathrm{r}\end{array}$ & 90 & 57.69 & 3.67 & 0.54 \\
\hline $\begin{array}{l}\text { Jatroph } \\
\text { a } \\
\text { gossypif } \\
\text { olia }\end{array}$ & $\begin{array}{l}\text { Lower } \\
\text { layer of } \\
\text { leaf }\end{array}$ & $\begin{array}{l}\text { Brachypara } \\
\text { cytic, } \\
\text { anomotetra } \\
\text { cytic }\end{array}$ & $\begin{array}{l}24 \text { or } \\
40\end{array}$ & 2.51 & 0.17 & $\begin{array}{l}\text { Irregula } \\
\mathrm{r}\end{array}$ & 180 & 115.38 & 7.40 & 2.25 \\
\hline $\begin{array}{l}\text { Jatroph } \\
\text { a } \\
\text { gossypif } \\
\text { olia } \\
\end{array}$ & Stem & $\begin{array}{l}\text { Anomocyti } \\
\text { c }\end{array}$ & 2 & 1.28 & 0.08 & $\begin{array}{l}\text { Rectan } \\
\text { gular }\end{array}$ & 200 & 128.25 & 8.22 & 0.89 \\
\hline $\begin{array}{l}\text { Jatroph } \\
\text { a } \\
\text { Gossypi } \\
\text { folia }\end{array}$ & Pedicel & $\begin{array}{l}\text { Anomocyti } \\
\text { c }\end{array}$ & 2 & 1.28 & 0.08 & $\begin{array}{l}\text { Rectan } \\
\text { gular }\end{array}$ & 200 & 128.25 & 8.22 & 0.89 \\
\hline $\begin{array}{l}\text { Jatroph } \\
\text { a } \\
\text { Gossypi } \\
\text { folia }\end{array}$ & Calyx & $\begin{array}{l}\text { Brachypara } \\
\text { cytic, } \\
\text { Anomotetr } \\
\text { acytic }\end{array}$ & $\begin{array}{l}24 \text { Or } \\
40\end{array}$ & 2.51 & 0.17 & $\begin{array}{l}\text { Irregula } \\
\text { r To } \\
\text { Rectan } \\
\text { gular }\end{array}$ & 180 & 115.38 & 7.40 & 2.25 \\
\hline $\begin{array}{l}\text { Jatroph } \\
\text { a } \\
\text { Gossypi } \\
\text { folia }\end{array}$ & Corolla & Not Found & & & & Oval & 120 & 76.93 & 4.93 & \\
\hline $\begin{array}{l}\text { Jatroph } \\
\text { a } \\
\text { Gossypi } \\
\text { folia }\end{array}$ & $\begin{array}{l}\text { Gynocei } \\
\text { um }\end{array}$ & Not Found & & & & $\begin{array}{l}\text { Irregula } \\
\mathrm{r}\end{array}$ & 1165 & 746.80 & 47.87 & \\
\hline $\begin{array}{l}\text { Jatroph } \\
\text { a } \\
\text { Gossypi } \\
\text { folia }\end{array}$ & $\begin{array}{l}\text { Androce } \\
\text { ium }\end{array}$ & Not Found & & & & Oval & 1600 & $\begin{array}{l}1025.6 \\
4\end{array}$ & 65.75 & \\
\hline $\begin{array}{l}\text { Jatroph } \\
\text { a } \\
\text { Gossypi } \\
\text { folia }\end{array}$ & $\begin{array}{l}\text { Fruit } \\
\text { Wall }\end{array}$ & $\begin{array}{l}\text { Anomotetr } \\
\text { acytic }\end{array}$ & 3 & 1.92 & 0.12 & $\begin{array}{l}\text { Irregula } \\
\text { r To } \\
\text { Rectan } \\
\text { gular }\end{array}$ & 144 & 92.96 & 5.96 & 1.97 \\
\hline
\end{tabular}


Epidermal Studies in Identification of Jatropha Species

\begin{tabular}{|c|c|c|c|c|c|c|c|c|c|c|}
\hline $\begin{array}{l}\text { Jatroph } \\
a \\
\text { Curcas }\end{array}$ & $\begin{array}{l}\text { Upper } \\
\text { Layer } \\
\text { Of Leaf }\end{array}$ & $\begin{array}{l}\text { Anomocyti } \\
\text { c, } \\
\text { Anomotetr } \\
\text { acytic }\end{array}$ & $\begin{array}{l}1 \text { Or } \\
\text { Rare }\end{array}$ & 0.32 & 0.02 & $\begin{array}{l}\text { Irregula } \\
\text { r To } \\
\text { Polygo } \\
\text { nal }\end{array}$ & 70 & 44.87 & 2.88 & 0.69 \\
\hline $\begin{array}{l}\text { Jatroph } \\
\text { a } \\
\text { Curcas }\end{array}$ & $\begin{array}{l}\text { Lower } \\
\text { Layer } \\
\text { Of Leaf }\end{array}$ & $\begin{array}{l}\text { Anomocyti } \\
\text { c, } \\
\text { Anomotetr } \\
\text { acytic }\end{array}$ & 7 & 4.49 & 0.29 & $\begin{array}{l}\text { Irregula } \\
\text { r To } \\
\text { Polygo } \\
\text { nal }\end{array}$ & 150 & 96.64 & 6.19 & 4.48 \\
\hline $\begin{array}{l}\text { Jatroph } \\
\text { a } \\
\text { Curcas }\end{array}$ & Stem & $\begin{array}{l}\text { Anomocyti } \\
\text { c, } \\
\text { Anomotetr } \\
\text { acytic }\end{array}$ & 1 & 0.64 & 0.04 & $\begin{array}{l}\text { Irregula } \\
\text { r To } \\
\text { Polygo } \\
\text { nal }\end{array}$ & 26 & 16.67 & 1.07 & 3.60 \\
\hline $\begin{array}{l}\text { Jatroph } \\
a \\
\text { Curcas }\end{array}$ & Pedicel & $\begin{array}{l}\text { Anomocyti } \\
\text { c, } \\
\text { Anomotetr } \\
\text { acytic }\end{array}$ & 1 & 0.64 & 0.04 & $\begin{array}{l}\text { Rectan } \\
\text { gular } \\
\text { To } \\
\text { Pentago } \\
\text { nal }\end{array}$ & 300 & 192.96 & 12.37 & 0.32 \\
\hline $\begin{array}{l}\text { Jatroph } \\
a \\
\text { Curcas }\end{array}$ & Calyx & Actinocytic & $\begin{array}{l}1 \text { Or } \\
2\end{array}$ & 0.96 & 0.06 & Sepal & 500 & 320.51 & 20.55 & 0.29 \\
\hline $\begin{array}{l}\text { Jatroph } \\
\text { a } \\
\text { Curcas }\end{array}$ & Corolla & Actinocytic & 6 & 3.85 & 0.25 & $\begin{array}{l}\text { Irregula } \\
\text { r To } \\
\text { Rectan } \\
\text { gular }\end{array}$ & 360 & 230.76 & 14.79 & 1.66 \\
\hline $\begin{array}{l}\text { Jatroph } \\
\text { a } \\
\text { Curcas }\end{array}$ & $\begin{array}{l}\text { Gynocei } \\
\text { um }\end{array}$ & Not Found & & & & $\begin{array}{l}\text { Ellipsoi } \\
\text { d }\end{array}$ & 400 & 250.64 & 16.07 & \\
\hline $\begin{array}{l}\text { Jatroph } \\
\text { a } \\
\text { Curcas }\end{array}$ & $\begin{array}{l}\text { Androce } \\
\text { ium }\end{array}$ & Not Found & & & & $\begin{array}{l}\text { Irregula } \\
\mathrm{r}\end{array}$ & $\begin{array}{l}240- \\
560\end{array}$ & 250.64 & 16.07 & \\
\hline $\begin{array}{l}\text { Jatroph } \\
\text { a } \\
\text { Curcas }\end{array}$ & $\begin{array}{l}\text { Fruit } \\
\text { Wall }\end{array}$ & $\begin{array}{l}\text { Paratetracy } \\
\text { tic }\end{array}$ & 1 & 0.64 & 0.04 & Oval & 150 & 96.15 & 6.16 & 0.65 \\
\hline
\end{tabular}

4.1.2. Chart of stomata index

Comparison of jatropha gossypifolia and jatropha curcas

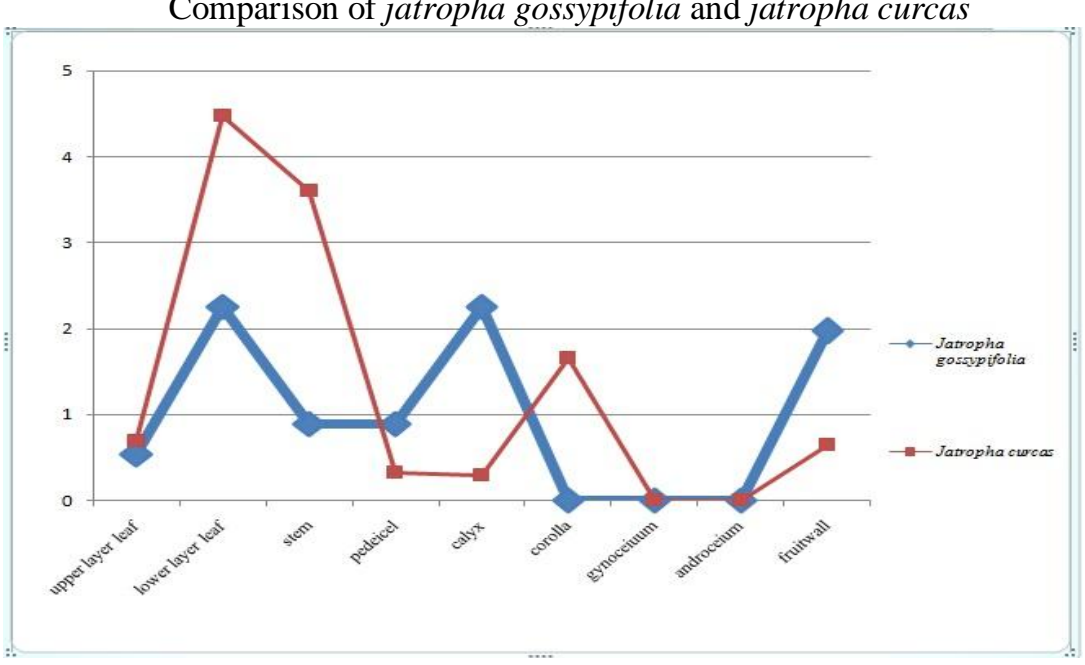


Epidermal Studies in Identification of Jatropha Species

\begin{tabular}{|c|c|c|c|c|c|c|c|c|c|c|}
\hline $\begin{array}{l}\text { Name of } \\
\text { plant }\end{array}$ & $\begin{array}{l}\text { Name of } \\
\text { plant } \\
\text { parts }\end{array}$ & $\begin{array}{l}\text { Type of } \\
\text { trichome }\end{array}$ & $\begin{array}{l}\text { Number } \\
\text { of } \\
\text { trichom } \\
\mathrm{e} \\
\end{array}$ & $\begin{array}{l}\text { frequenc } \\
y \text { of } \\
\text { trichome }\end{array}$ & $\begin{array}{l}\text { density } \\
\text { of } \\
\text { trichom } \\
\mathrm{e} \\
\end{array}$ & $\begin{array}{l}\text { Type of } \\
\text { epidermal } \\
\text { cell }\end{array}$ & $\begin{array}{l}\text { Number } \\
\text { of } \\
\text { epiderm } \\
\text { al cell } \\
\end{array}$ & $\begin{array}{l}\text { Frequenc } \\
y \quad \text { of } \\
\text { epiderma } \\
1 \text { cell }\end{array}$ & $\begin{array}{l}\text { Density } \\
\text { of } \\
\text { epiderm } \\
\text { al cell } \\
\end{array}$ & $\begin{array}{l}\text { Inde } \\
\mathrm{x} \text { of } \\
\text { trich } \\
\text { ome }\end{array}$ \\
\hline $\begin{array}{l}\text { Jatropha } \\
\text { gossypifolia }\end{array}$ & $\begin{array}{l}\text { Upper } \\
\text { layer of } \\
\text { leaf }\end{array}$ & $\begin{array}{l}\text { Uniseriate } \\
\text { flagellate } \\
\text { glandular } \\
\text { hair and } \\
\text { multiseriat } \\
\text { e capitate } \\
\text { glandular } \\
\text { hair }\end{array}$ & $\begin{array}{ll}1 & \text { or } \\
\text { rare } & \end{array}$ & .32 & 0.02 & irregular & 90 & 57.69 & 3.67 & 0.54 \\
\hline $\begin{array}{l}\text { Jatropha } \\
\text { gossypifolia }\end{array}$ & $\begin{array}{l}\text { Lower of } \\
\text { leaf }\end{array}$ & $\begin{array}{l}\text { Uniseriate } \\
\text { flagellate } \\
\text { glandular } \\
\text { hair and } \\
\text { multiseriat } \\
\text { e capitate } \\
\text { glandular } \\
\text { hair }\end{array}$ & $\begin{array}{ll}12 & \text { or } \\
\text { rare } & \end{array}$ & 3.85 & 0.25 & irregular & 180 & 115.38 & 7.40 & 3.27 \\
\hline $\begin{array}{l}\text { Jatropha } \\
\text { gossypifolia }\end{array}$ & Stem & $\begin{array}{l}\text { Uniseriate } \\
\text { flagellate } \\
\text { glandular } \\
\text { hair and } \\
\text { multiseriat } \\
\text { e capitate } \\
\text { glandular } \\
\text { hair }\end{array}$ & 5 & 3.21 & 0.21 & $\begin{array}{l}\text { rectangula } \\
\mathrm{r}\end{array}$ & 200 & 128.25 & 8.22 & 2.49 \\
\hline $\begin{array}{l}\text { Jatropha } \\
\text { gossypifolia }\end{array}$ & Pedicel & $\begin{array}{l}\text { Uniseriate } \\
\text { flagellate } \\
\text { glandular } \\
\text { hair and } \\
\text { multiseriat } \\
\text { e capitate } \\
\text { glandular } \\
\text { hair }\end{array}$ & 5 & 3.21 & 0.21 & $\begin{array}{l}\text { rectangula } \\
\mathrm{r}\end{array}$ & 200 & 128.25 & 8.22 & 2.49 \\
\hline $\begin{array}{l}\text { Jatropha } \\
\text { gossypifolia }\end{array}$ & Calyx & $\begin{array}{l}\text { Uniseriate } \\
\text { flagellate } \\
\text { glandular } \\
\text { hair and } \\
\text { multiseriat } \\
\text { e capitate } \\
\text { glandular } \\
\text { hair }\end{array}$ & 12 & 7.71 & 0.49 & $\begin{array}{l}\text { Irregular } \\
\text { to } \\
\text { rectangula } \\
\mathrm{r}\end{array}$ & 180 & 115.38 & 7.40 & 6.21 \\
\hline $\begin{array}{l}\text { Jatropha } \\
\text { gossypifolia }\end{array}$ & Corolla & $\begin{array}{l}\text { Uniseriate } \\
\text { flagellate } \\
\text { glandular } \\
\text { hair and } \\
\text { multiseriat } \\
\text { e capitate } \\
\text { glandular } \\
\text { hair }\end{array}$ & 3 or 5 & 2.56 & 0.16 & oval & 120 & 76.93 & 4.93 & 3.14 \\
\hline $\begin{array}{l}\text { Jatropha } \\
\text { gossypifolia }\end{array}$ & $\begin{array}{l}\text { Gynocei } \\
\text { um }\end{array}$ & Not found & & & & irregular & 1165 & 746.80 & 47.87 & \\
\hline $\begin{array}{l}\text { Jatropha } \\
\text { gossypifolia }\end{array}$ & $\begin{array}{l}\text { Androcei } \\
\text { um }\end{array}$ & $\begin{array}{l}\text { Uniseriate } \\
\text { flagellate } \\
\text { glandular } \\
\text { hair and } \\
\text { multiseriat } \\
\text { e capitate } \\
\text { glandular } \\
\text { hair }\end{array}$ & 1 & .64 & 0.04 & oval & 1600 & 1025.64 & 65.75 & 0.06 \\
\hline $\begin{array}{l}\text { Jatropha } \\
\text { gossypifolia }\end{array}$ & fruit wall & $\begin{array}{l}\text { Uniseriate } \\
\text { flagellate } \\
\text { glandular } \\
\text { hair and } \\
\text { multiseriat } \\
\text { e capitate } \\
\text { glandular } \\
\text { hair }\end{array}$ & 4 & 2.56 & 0.16 & $\begin{array}{l}\text { Irregular } \\
\text { to } \\
\text { rectangula } \\
\mathrm{r}\end{array}$ & 144 & 92.96 & 5.96 & 2.61 \\
\hline $\begin{array}{l}\text { Jatropha } \\
\text { curcas }\end{array}$ & $\begin{array}{l}\text { Upper } \\
\text { layer of } \\
\text { leaf }\end{array}$ & $\begin{array}{l}\text { Uniseriate } \\
\text { capitates } \\
\text { glandular }\end{array}$ & $\begin{array}{ll}1 & \text { or } \\
\text { rare }\end{array}$ & .32 & 0.02 & $\begin{array}{l}\text { Irregular } \\
\text { to } \\
\text { polygonal }\end{array}$ & 70 & 44.87 & 2.88 & 0.69 \\
\hline
\end{tabular}


Epidermal Studies in Identification of Jatropha Species

\begin{tabular}{|c|c|c|c|c|c|c|c|c|c|c|}
\hline & & $\begin{array}{l}\text { hair, } \\
\text { Uniseriate } \\
\text { flagellate } \\
\text { glandular } \\
\text { hair }\end{array}$ & & & & & & & & \\
\hline $\begin{array}{l}\text { Jatropha } \\
\text { curcas }\end{array}$ & $\begin{array}{l}\text { Lower of } \\
\text { leaf }\end{array}$ & Not found & $\begin{array}{ll}1 & \text { or } \\
\text { rare } & \end{array}$ & .32 & 0.02 & $\begin{array}{l}\text { Irregular } \\
\text { to } \\
\text { polygonal }\end{array}$ & 150 & 96.64 & 6.19 & 0.32 \\
\hline $\begin{array}{l}\text { Jatropha } \\
\text { curcas }\end{array}$ & Stem & $\begin{array}{l}\text { Unicelled } \\
\text { flagellate } \\
\text { glandular } \\
\text { hair. }\end{array}$ & $1,4,3$ & 1.71 & 0.11 & $\begin{array}{l}\text { Irregular } \\
\text { to } \\
\text { polygonal }\end{array}$ & 26 & 16.67 & 1.07 & 9.32 \\
\hline $\begin{array}{l}\text { Jatropha } \\
\text { curcas }\end{array}$ & Pedicel & $\begin{array}{l}\text { Uniseriate } \\
\text { capitate } \\
\text { glandular } \\
\text { hair, } \\
\text { developin } \\
\mathrm{g} \\
\text { uniseriate } \\
\text { flagellate } \\
\text { glandular } \\
\text { hair, } \\
\text { unicelled } \\
\text { branched } \\
\text { glandular } \\
\text { hair, } \\
\text { papillate } \\
\text { glandular } \\
\text { hair }\end{array}$ & $\begin{array}{ll}1 & \text { or } \\
\text { rare } & \end{array}$ & .32 & 0.02 & $\begin{array}{l}\text { Rectangul } \\
\text { ar to } \\
\text { pentagonal }\end{array}$ & 300 & 192.96 & 12.37 & 0.16 \\
\hline $\begin{array}{l}\text { Jatropha } \\
\text { curcas }\end{array}$ & Calyx & $\begin{array}{l}\text { Uniseriate } \\
\text { flagellate } \\
\text { glandular } \\
\text { hair } \\
\text { unicelled } \\
\text { flagellate } \\
\text { non } \\
\text { glandular } \\
\text { hair, } \\
\text { unicelled } \\
\text { branched } \\
\text { glandular } \\
\text { hair }\end{array}$ & $\begin{array}{ll}1 & \text { or } \\
\text { rare } & \end{array}$ & .32 & 0.02 & sepal & 500 & 320.51 & 20.55 & 0.10 \\
\hline $\begin{array}{l}\text { Jatropha } \\
\text { curcas }\end{array}$ & Corolla & $\begin{array}{l}\text { Not } \\
\text { found }\end{array}$ & 1 & .64 & 0.04 & $\begin{array}{l}\text { Irregular } \\
\text { to } \\
\text { rectangula } \\
\mathrm{r}\end{array}$ & 360 & 230.76 & 14.79 & 0.27 \\
\hline $\begin{array}{l}\text { Jatropha } \\
\text { curcas }\end{array}$ & $\begin{array}{l}\text { Gynocei } \\
\text { um }\end{array}$ & $\begin{array}{l}\text { Uniseriate } \\
\text { capitate } \\
\text { glandular } \\
\text { hair, } \\
\text { unicelled } \\
\text { branched } \\
\text { glandular } \\
\text { hair }\end{array}$ & 1 & .64 & 0.04 & ellipsoid & 400 & 250.64 & 16.07 & 0.25 \\
\hline $\begin{array}{l}\text { Jatropha } \\
\text { curcas }\end{array}$ & $\begin{array}{l}\text { Androcei } \\
\text { um }\end{array}$ & $\begin{array}{l}\text { Unicelled } \\
\text { flagellate } \\
\text { non } \\
\text { glandular } \\
\text { hair }\end{array}$ & 2,6 & 2.56 & 0.16 & irregular & $240-560$ & 250.64 & 16.07 & 0.99 \\
\hline $\begin{array}{l}\text { Jatropha } \\
\text { curcas }\end{array}$ & fruit wall & $\begin{array}{l}\text { Uniseriate } \\
\text { capitate } \\
\text { glandular } \\
\text { hair } \\
\text { unicelled } \\
\text { branched } \\
\text { glandular } \\
\text { hair }\end{array}$ & 1 & .64 & 0.04 & oval & 150 & 96.15 & 6.16 & 0.65 \\
\hline
\end{tabular}


4.1.4. Chart of trichome index

Comparison of jatropha gossypifolia and jatropha curcas

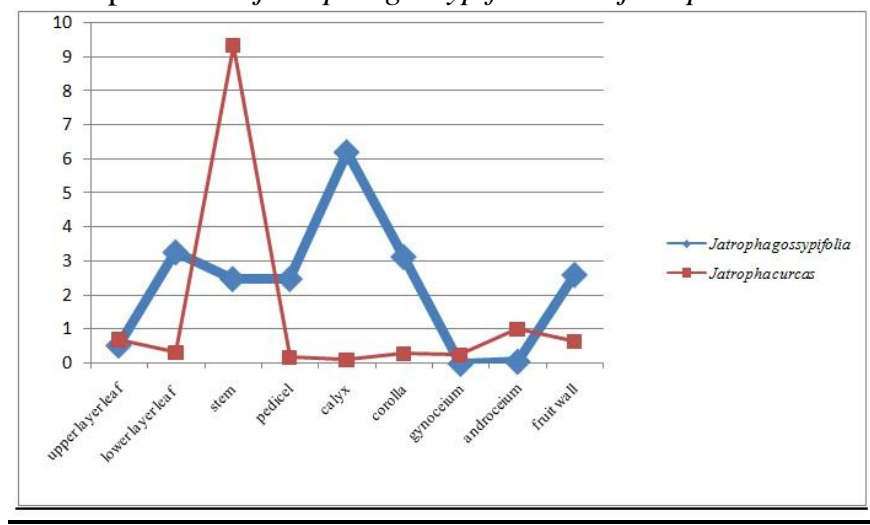

4.1.5. Trichome Plate A

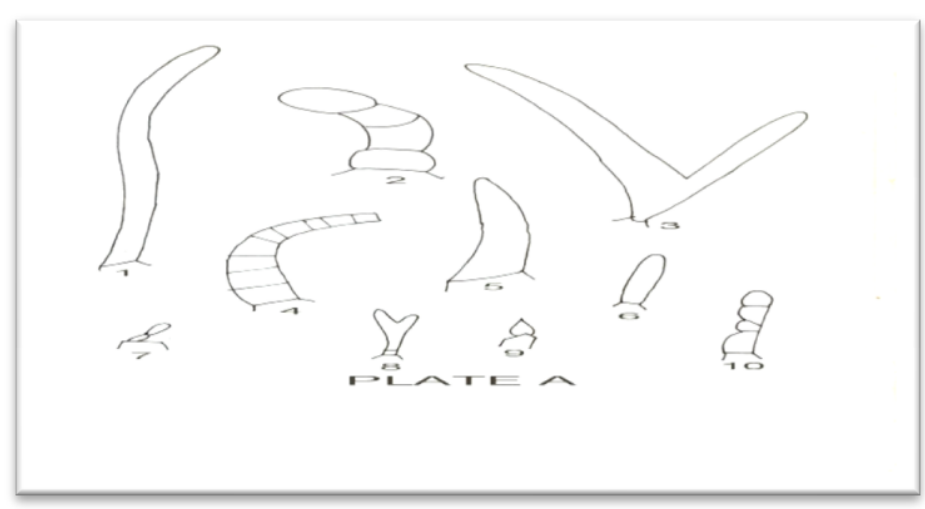

4.1.6. In jatropha curcas trichome plate A

Fig.1, 5, and 6: Unicelled flagellate glandular hair

Fig. 1: 40X87.50

Fig.5: 40X38.75

Fig.6: 40X12.50

Fig.2: Uniseriate capitates glandular hair 40X31.25

Fig.7: Developing uniseriate flagellate glandular hair 10x30

Fig.4, 10: Uniseriate flagellate glandular hair

Fig.4: 40X25

Fig. 10:10X100

Fig.3, 8: Unicelled branched glandular hair

Fig.3: $10 \times 250$

Fig.8: 10X85

Fig.9: Papillate glandular hair 10X25

4.1.7. Trichome plate B

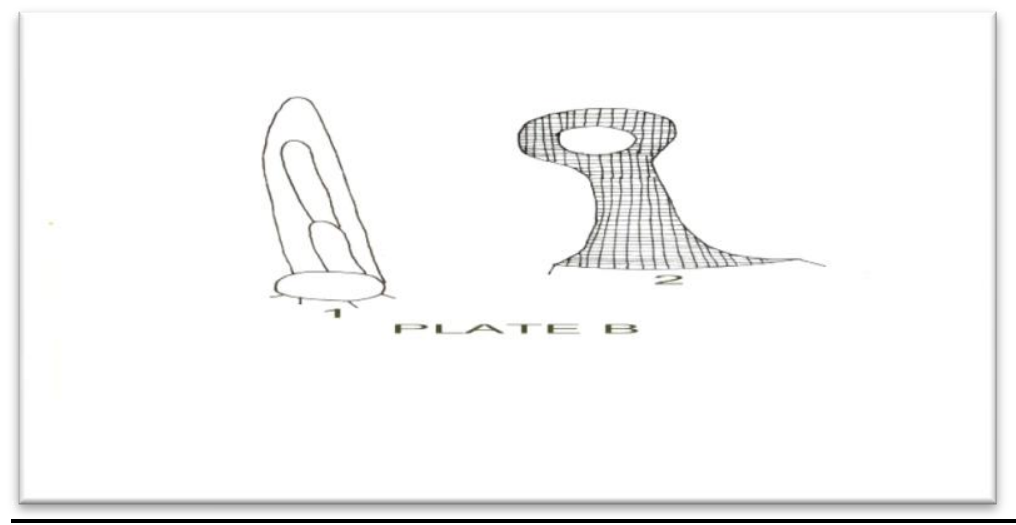


4.1.8. In jatropha gossypifolia trichome plate B

Fig.1: Uniseriate flagellate glandular hair 40X75

Fig.2: Multiseriate capitates glandular hair 10X350

4.1.9. Stomata plate A

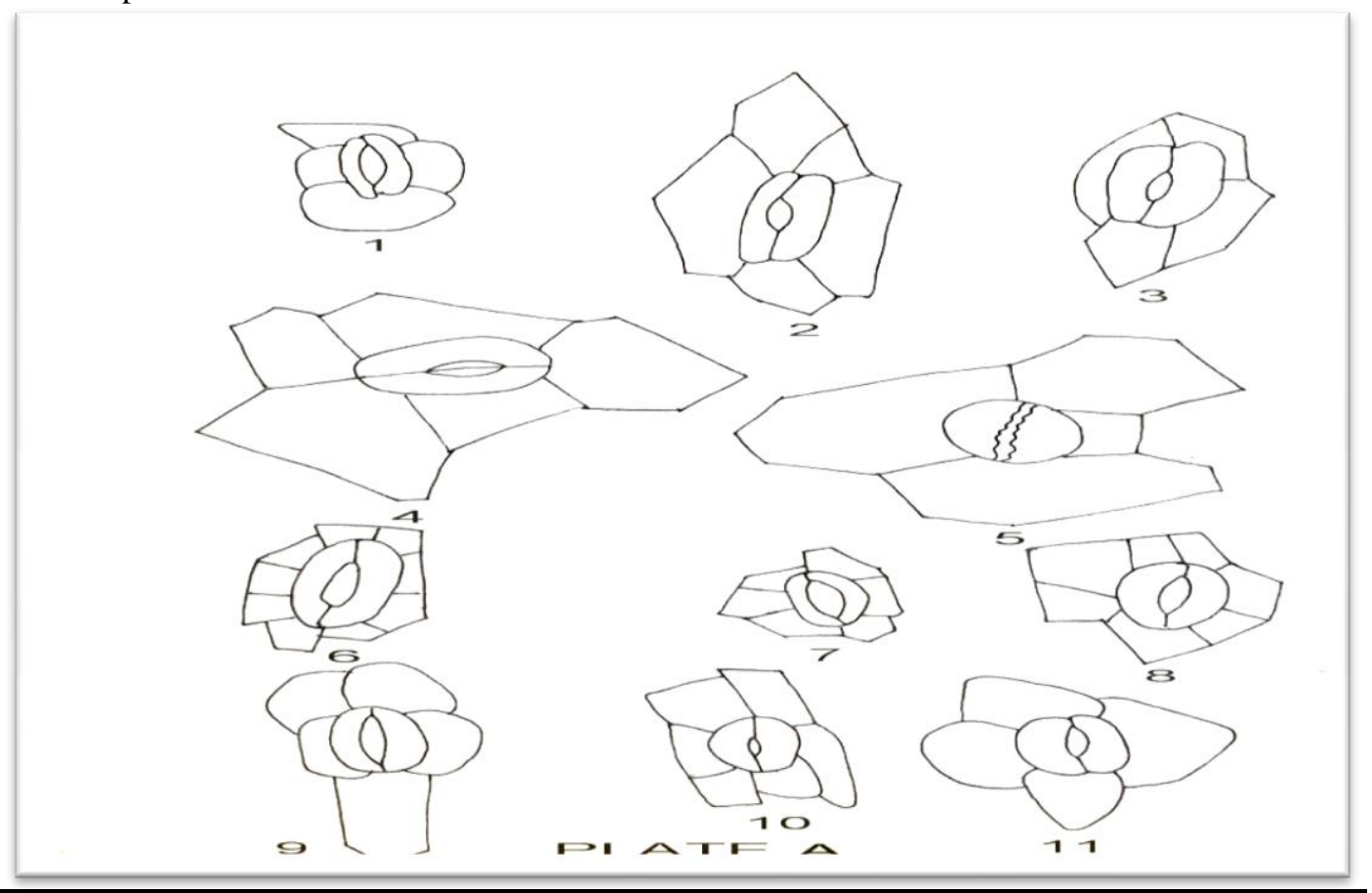

4.1.10. In jatropha curcas stomata plate $\mathrm{A}$

Fig.1: Paratetracytic stomata, in fruit wall,

Size: in $40 \mathrm{X}$

Stomata: $1=12.50, b=8.75$

Stomata with subsidery cell: $1=25, b=18.75$

Fig. 2, 4, 6: Anomocytic stomata

Fig.4: in leaf

Size: in $40 \mathrm{X}$

Stomata: $1=15, b=12.50$

Stomata with subsidery cell: $1=38.75, b=26.25$

Fig.2 in stem

Size: in 40X

Stomata: $1=18.75, b=11.25$

Stomata with subsidery cell: $1=47.50, b=18.75$

Fig. 6 in pedicel

Size: in 40X

Stomata: $1=18.75, b=7.50$

Stomata with subsidery cell: $1=25, b=15$

Fig.3, 5: Anomotetracytic stomata

Fig.5: in leaf

Size: in 40X

Stomata: $1=18.75, b=12.50$

Stomata with subsidery cell: $1=56.25, b=25$

Fig.3: in stem

Size: in 40X

Stomata: $\mathrm{l}=22.50, \mathrm{~b}=10$

Stomata with subsidery cell: $1=50, b=21.25$

Fig.7, 8: Actinocytic stomata

Fig.7: in sepal

Size: in $40 X$

Stomata: $1=12.50, b=10$

Stomata with subsidery cell: $l=18.75, b=15$

Fig.8: in petal 
Size: in $40 \mathrm{X}$

Stomata: $l=13.75, b=11.25$

Stomata with subsidery cell: $1=22.5, b=18.75$

4.1.11. In jatropha gossypifolia stomata plate A

Fig.9: Brachyparacytic stomata, in leaf

Size: in $40 \mathrm{X}$

Stomata: $1=12.50, b=8.75$

Stomata with subsidery cell: $1=31.25, b=18.75$

Fig.10: Anomocytic stomata, in stem

Size: in $40 \mathrm{X}$

Stomata: $1=12.50, b=8.75$

Stomata with subsidery cell: $l=30, b=16.25$

Fig. 11: Anomotetracytic stomata, in fruit wall

Size: in $40 \mathrm{X}$

Stomata: $l=12.50, b=12.50$

Stomata with subsidery cell: $1=25, b=27.5$

\section{Results and discussion}

Jatropha curcas and Jatropha gossypifolia belongs to Euphorbiaceous family. They are found in tropical regions. Jatropha curcas is perennial plant. It has pale green leaves, erect stem, male and female flowers and ellipsoid capsule. It has many toxic contents curcin A and B, phorbolesters. It is useful in treatment of many diseases. Its toxicity can be showed in form of diarrhea, posture, depression. Jatropha gossypifolia is busy shrub. It has lobed, wide leaves, hairy, non-woody stem, red crimson flowers in corymbs inflorescence and oblong capsule. It has toxic content ricinine and jatrophin. It is used for toothache, laprosy, ulcers, skin itches etc. When it consumed in excess then it produce abdominal pain, ptosis, and hind-limb paralysis.

There has been found brachyparacytic, anomotetracytic stomata in Jatropha gossypifolia and anomocytic, anomotetracytic, actinocytic, paratetracytic stomata in Jatropha curcas. The largest stomata 24 or 40 have been found in calyx and lower layer of leaf of Jatropha gossypifolia. The largest stomatal frequency 4.49 has been found in lower layer of leaf of Jatropha curcas. The largest stomatal density 0.29 has been found in lower layer of leaf of Jatropha curcas. The largest epidermal number 1600 has been found in androceium of Jatropha gossypifolia. The largest frequency of epidermal cell 1025.64 has been found in androceium of Jatropha gossypifolia. The largest density of epidermal cell 65.75 has been in androceium of Jatropha gossypifolia. The largest stomatal index 4.48 has been found in lower layer of leaf of Jatropha curcas. Stomata, stomatal frequency, stomatal density has been absent in corolla, gynoceium, androceium of Jatropha gossypifolia and in gynoceium, androceium of Jatropha curcas. The lowest epidermal number 26 has been found in stem of Jatropha curcas. The lowest epidermal frequency 16.67 has been found in stem of Jatropha curcas. The lowest epidermal density 1.07 has been found in stem of Jatropha curcas. There has been found uniseriate aseptate flagellate glandular hair, multiseriate capitate glandular hair in Jatropha gossypifolia and bicelled uniseriate glandular hair, uniseriate glandular hair, uniseriate sekile shaped glandular hair, simple two armed cylindrical glandular hair, acinaciform non glandular hair, cylindrical non glandular hair in Jatropha curcas. The largest trichome number 12 has been found in calyx of Jatropha gossypifolia. The largest trichome frequency 7.71 has been found in calyx of Jatropha gossypifolia. The largest trichome density 0.49 has been found in calyx of Jatropha gossypifolia. The largest trichome index 9.32 has been found in stem of Jatropha curcas. There have been absent trichome, trichome index, trichome frequency, trichome density in gynoceium of Jatropha gossypifolia.

\section{Conclusion}

Economically Jatropha curcas and Jatropha gossypifolia are useful that included in Euphorbiaceous family. As well as they are showed also toxic properties. They can be identified by brachyparacytic, anomotetracytic, anomocytic, actinocytic, paratetracytic stomata, uniseriate flagellate glandular hair, multiseriate capitate glandular hair, uniseriate capitate glandular hair, unicelled flagellate eglandular hair, uniseriate flagellate glandular hair, unicelled branched glandular hair, papillate glandular hair and other data parameters in the taxonomy.

\section{Acknowledgement}

"One touch of nature makes the whole work kin"

Apart from our hard work, devotion and grace of our parents the completion of this research required a helping hand of number of person, here is acknowledge in the honor of those super persons. First of all our 
sincere thanks to respected Dr. Ela Tiwari without whose kind permission the project would have not even started, also the lecturer of Botany department who guided us all the way through this research.

We should be failing duly if we do not express out deep sense of gratitude to all our friends who have shared their valuable time and helped us directly or indirectly in the preparation of our research.

At this end, last but not least we would like to thanks to other staff members of this department.

\section{Journal papers:}

\section{References}

[1] Abdulrahaman, A.A., Egbedo F.O., Oladele, F.A.2009. Stomatal complex types, stomatal density and the stomatal index in some species of Dioscorea. Archives of biological sciences, vol. 61(4), pp.847-851.

[2] Abdulrahaman, A.A., Egbedo F.O., Oladele, F.A.2010. Stomatal complex types, stomatal density and the stomatal index in some Jatropha species L. (Euphorbiaceae). Nig.J.Pure\&Appl. Sci. Vol. 23; 2160-2163.

[3] Patel, R. C. And J. A. Inamdar, 1970. Structure and ontogeny of stomata in some polemoniales. Oxford Journals, Life sciences, Annals of Botany Vol. 35(2) pp.389-409.

[4] Fadeyi, A., Adeoye, A.O. and Olowokundejo, J. D.1989. Epidermal and phytochemical studies in the genus Boerhavia (Nyctaginceae) in Nigeria, International J. Crude drug Res 27: 178-184.

[5] Camargo, M.A.B. and R.A. Marenco. 2011. Density size and distribution of stomata in 35 rainforest tree species in Central Amazonia. Acta Amaz. Vol 41(2).

[6] Hameed, I., F. Hussain and G. Dastagir.2008. Stomatal studies of some selected medicinal plants of Poligonaceae. Pak.J.Bot, 40(6):2213-2280.

[7] Abid, R., Sharmeen, S. And Perveen, A.2007.Stomatal types of monocots within flora of Karachi, Pakistan.Pak.J.Bot. 39(I): 15:$27,2007$.

[8] Ahmad, K., Khan, M.A., Ahmad, M., Zafar, M., Arshad, M., and Ahmad, F.2009. Taxonomic diversity of stomata in dicot flora of a district tank (N.W.F.P.) in Pakistan. African journal of Biotechnology vol. 8(6); 1052:-1055.

[9] Ramayya, N.1962b. Studies on the trichome of some Composite I. General structure. Bull. Bot. Serv. India, 1:-4:187:-192.

[10] Sahu, T.R.1982. Studies on the trachoma's in Helianthoideae (Asteraceae). J.Econ.Tax.Bot.Vol.3:517:-521.

[11] Sahu, T.R.1983. Trichome studies in senecio linn: structure, distribution and Taxonomic significance. I. Indian Bot. Soc.62:84:-89.

[12] Sahu, T.R.1984.Taxonomic implications of trichome complements to Vernonia (Compositae) in India.Feddes Repertorium, 95(4):237:-249.

[13] Sahu, T.R.1985. Studies on the trichomes in Asteroideae (Asteraceae). Proc.Nat.Acad.Sci.India, 55(B), I: 39:-44.

[14] Tiwari, E. And T. R. Sahu, 1982. Taxonomic implication of trichome morphology in pluchea cass. (Compositae). Life Science Advance, I (3):315:-317.

[15] Faust, W. Z. And Jr. S. B. Johnes. 1973. The systematic value of trichome complements in North American group of Vernonia (Compositae). Rhodora, 75:517-528.

[16] Inamdar, J.A. and M.Gangadhara, 1976. Structure, ontogeny, classification and taxonomic significance of stomata in Cucurbitaceae. Feddes Repertorium, 87(5):293-310.

[17] Boubos, S. T. And A.B. Beakbane.1971. A chemical method for separating leaf epidermis from mesophyll tissue. U.A.R. J.Bot., 17:317-322.

[18] Salisbury, E.J.1927. On the causes and ecological significance of stomatal frequency, with special reference to the woodland flora. Philos. Trans. R. Soc. London. B: 216, 1-65.

[19] Payne, W.W. 1978. A glossary of plant hair terminology Brittonia. 30, 239-255.

[20] Dilcher, D.L.1974. Approaches to the identification of angiosperm leaf remains.Bot. Rev. 40:1-157.

[21] Books:

[22] Iwu, M.M.1993. Handbook of African medicinal plants. (CRC Press, New York pp. 194).

[23] Smith, A.B.1923. Poisonous plants of all countries 2nd eds, (Tin dall and Company, London).

[24] Champion, H.G. and S. K. Seth 1968. A revised survey of the forest types of India (Delhi XXVII, 404 P).

[25] Metcalfe, C. R. And L. Chalk. 1950. Anatomy of the dicotyledons, vol. I (Clarendon press).

[26] Satce, C. A. 1984. Plant taxonomy and biosystematics (Cambridge University Press).

[27] Cutler, D.F., T. Botha, C.E.J. Botha, D.W. Stevenson 2008. Plant anatomy: An applied approach (John wiley \&Sons Pp.302)

[28] Proceeding paper:

[29] Perveen, A., Abid, R., and Fatima, R.2007.Stomatal types of some dicots within flora of Karachi, Pakistan. Pak.J.Bot. 39(4):1 017:1023. 